\section{RMD Open}

Rheumatic \&

Musculoskeletal Diseases
To cite: Druce KL, Iqbal K, Watson KD, et al. Mortality in patients with interstitial lung disease treated with rituximab or TNFi as a first biologic. RMD Open 2017;3:e000473. doi:10.1136/ rmdopen-2017-000473

- Prepublication history for this paper is available online. To view these files please visit the journal online (http://dx.doi. org/10.1136/rmdopen-2017000473).

Received 3 April 2017 Revised 18 May 2017 Accepted 6 June 2017

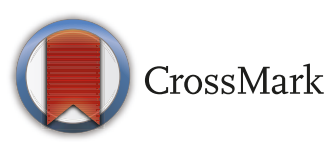

${ }^{1}$ Arthritis Research UK Centre for Epidemiology, Institute of Inflammation and Repair, University of Manchester, Manchester Academic Health Science Centre, Manchester, UK 2Department of Rheumatology, Queen Elizabeth Hospital, Gateshead, UK

${ }^{3}$ National Institute of Health Research Manchester Musculoskeletal Biomedical Research Unit, Central Manchester Foundation Trust and University of Manchester, Manchester Academic Health Science Centre, Manchester, UK

Correspondence to Clive Kelly; katie.druce@ manchester.ac.uk

\title{
Mortality in patients with interstitial lung disease treated with rituximab or TNFi as a first biologic
}

Katie L Druce, ${ }^{1}$ Kundan Iqbal, ${ }^{2}$ Kath D Watson, ${ }^{1}$ Deborah P M Symmons, ${ }^{1,3}$ Kimme L Hyrich, ${ }^{2}$ Clive Kelly ${ }^{1}$

\section{ABSTRACT}

Objectives Guidelines cautioned prescribing of tumour necrosis factor inhibitors (TNFi) to patients with rheumatoid arthritis and interstitial lung disease (RA-ILD) after reports of new or worsening of ILD. Less is known about outcomes among patients with RA-ILD who receive rituximab (RTX). This study compares mortality in patients with RA-ILD who received RTX or TNFi as their first biologic.

Methods Participants with RA-ILD recruited to the British Society for Rheumatology Biologics Register for RA were included. Death rates were calculated and risk comparisons were made using Cox regression. Causes of death, including the frequency in which ILD was recorded on death certificates were examined.

Results 43 patients on RTX and 309 on TNFi were included. RTX recipients had shorter disease duration and less disability. Death rates were 94.8 (95\% Cl: 74.4 to 118.7) and 53.0 (22.9 to 104.6) per 1000 person years, respectively. The adjusted mortality risk was halved in the RTX cohort, but the difference was not statistically significant (HR 0.53, 95\% Cl: 0.26 to 1.10). ILD was the underlying cause of death in 1 of 7 RTX deaths (14\%) and 12 of 76 TNFi deaths (16\%).

Conclusions Patients with RA-ILD who received RTX had lower mortality rates compared to TNFi. The absence of information on ILD severity or subtype prevents conclusions of which drug represents the best choice in patients with RA-ILD and active arthritis.

\section{BACKGROUND}

Pulmonary involvement, including interstitial lung disease (ILD), is common in patients with rheumatoid arthritis (RA). ${ }^{12}$ Such extra-articular manifestations are widely recognised to be associated with increased mortality. ${ }^{1-4}$ Despite overall mortality falling in recent decades in patients with RA, RA-ILD mortality rates appear to be increasing. ${ }^{45}$

The treatment of active arthritis in patients with RA-ILD is challenging due to concerns about exacerbation of lung disease with certain conventional synthetic disease-modifying antirheumatic drugs (csDMARDs) ${ }^{6}$ and tumour necrosis factor inhibitors (TNFi). ${ }^{7}$

\section{Key messages}

What is already known about this subject?

- Concerns about the use of some biologics make managing active arthritis in patients with rheumatoid arthritis and interstitial lung disease (RA-ILD) particularly challenging.

- Compared with a wealth of data regarding the link between RA-ILD and tumour necrosis factor inhibitors (TNFi), less is known regarding the outcomes and mortality risk in patients with RA-ILD who receive rituximab (RTX).

What does this study add?

- We have shown that patients who selected to receive RTX appear to have better long-term survival than those who receive TNFi as their first biologic.

- We also demonstrate that most of the deaths observed were not attributed to ILD.

How might this impact on clinical practice?

- This manuscript provides a crucial step towards determining the best recommendations for the management of active arthritis in this population.

Indeed, the British Society for Rheumatology (BSR) has specifically cautioned prescribing TNFi to patients with RA-ILD. ${ }^{5}$ Rituximab (RTX), which has been successfully used in the treatment of refractory ILD, ${ }^{8}$ may be considered as an alternative biologic in these patients. ${ }^{9}$ However, ILD has been reported as a complication of treatment in lymphoma patients treated with $\mathrm{RTX}^{1011}$ and less is known regarding the outcomes and mortality risk in patients with RA-ILD who receive RTX. $^{12-14}$

Previous analyses within the British Society for Rheumatology Biologics Register for RA (BSRBR-RA) ${ }^{15}$ reported no significant difference in mortality rates between patients with RA-ILD treated with TNFi or csDMARDs. However, a greater proportion of deaths were attributed to RA-ILD in the TNFi cohort. This 
current study aims to investigate 5-year mortality rates and causes of death among patients with RA-ILD treated with either RTX or TNFi as their first-line biologic therapy.

\section{METHODS}

The BSRBR-RA is a national, prospective, observational cohort study which has recruited patients with RA commencing a biologic therapy in order to examine the long-term safety of these medications. ${ }^{1617}$ Patients starting a TNFi (infliximab (Remicade), etanercept (Enbrel) or adalimumab (Humira)) were recruited between 2001 and 2007 and again from 2010, at which point the register opened again to recruit patients starting these three original TNFi as well as certolizumab pegol (Cimzia). Patients starting RTX were actively recruited between beginning of 2008 and 30 September 2011, during which patients starting RTX as either a first-line or second-line drug were registered.

Participants eligible for this analysis had physician's diagnosed RA-ILD ('Has the patient ever had pulmonary fibrosis?') and were recruited prior to 30 September 2011 (to allow a full 5 years of follow-up at the point of data analyses), at the point of commencing either RTX (Mabthera) or a TNFi: infliximab (Remicade), etanercept (Enbrel) or adalimumab (Humira) as their first biologic therapy and had returned at least one follow-up form.

\section{Data}

Baseline data collected included sex, age, ethnicity, smoking history, disease duration, disease activity, measured using the 28-joint count disease activity score, ${ }^{18}$ current and past csDMARDs, corticosteroid use and comorbidities including presence of asthma and chronic obstructive pulmonary disease (COPD) and disability (Stanford Health Assessment Questionnaire (HAQ) ). ${ }^{19}$ The primary outcome measure for this analysis was death, which was captured by: (1) rheumatologist follow-up questionnaire (returned 6 or 12 month follow-up questionnaire depending on time since treatment commenced), (2) family report to the register and (3) 'flagging' with the UK Office for National Statistics, which provided a copy of the death certificate, including cause of death. Reporting of death, including cause of death, is a mandatory requirement for all deaths occurring in the UK. Secondary outcomes included (1) the proportion of deaths which listed RA-ILD as the underlying cause of death and (2) the proportion of deaths which listed RA-ILD anywhere on the death certificate. RA-ILD was identified using International Classification of Diseases-10 codes J84.1 'interstitial pulmonary disease with fibrosis' and J84.9 'interstitial pulmonary disease, unspecified' and M05.1 'rheumatoid lung'.

\section{Statistical analysis}

Baseline characteristics were compared between the cohorts, using Pearson $\chi^{2}$ tests and Student's t-tests. Years of follow-up time were calculated from the date of starting biologics until death, or 5 years following first registration, whichever came first. Mortality rates, per 1000 person years (pyrs) with 95\% CI were calculated using an ever-exposed model, assuming a Poisson distribution of cases. Kaplan-Meier survival curves for mortality were generated. The risk of death between cohorts was compared using Cox regression. Confounders taken forward to a fully adjusted model included the $a$ priori selected variables age, sex and disease duration. Further any additional factors which shifted the unadjusted risk of mortality between RTX and TNFi by more than $10 \%$ were included as confounders. Additional candidate confounders included ethnicity, smoking history, disability (measured using the HAQ), disease activity (measured using the DAS28 (disease activity score-28)) previous DMARD and current steroid use, number of comorbidities and diagnoses of asthma and COPD. Missing data were accounted for in variables of interest using multiple imputation, with 20 imputed datasets. The variables on which multiple imputation was conducted and the proportion of missing data are shown in table 1 . Variables included in the imputation model included those offered as candidate confounders, the natural log of follow-up time and death.

All analyses were conducted using Stata V.13.1 (StataCorp).

\section{RESULTS}

Of 1632 recruited to the RTX and 15644 participants recruited to the TNFi cohort by 30 September 2011, 352 patients (43 RTX (6.8\%) and 309 TNFi $(2.0 \%)$ ) satisfied criteria for inclusion in the analysis.

Age and gender were comparable in the two cohorts (table 1). The mean disease activity using the 28-joint count disease activity score (DAS28), of those commencing RTX was lower than that of those commencing TNFi (mean: 6.3 (SD:1.1) vs 6.6 (1.0)). Those commencing RTX had significantly shorter median disease duration (median: 5.5 (IQR 3-13) vs 12 years $(7-20))$, had, on average, used fewer previous csDMARDs (3.2 (1.2) vs $4.0(1.6))$ and had less disability (HAQ: $1.6(0.7)$ vs $2.1(0.5)$ ) than patients starting TNFi. All patients commencing RTX did so after 2008 and all patients commencing TNFi did so prior to 2008 and thus there was no overlap in patient recruitment. Finally, the prevalence of pulmonary comorbidities was significantly greater in the RTX cohort (41.9\%) compared with the TNFi cohort $(25.6 \%, \mathrm{p}<0.03)$, driven by the presence of COPD (RTX: $33.3 \%$ ( $n=14)$ vs TNFi: $18.8 \%(n=58)$, $\mathrm{p}<0.03)$.

During 801.3 pyrs, 76 deaths occurred in the TNFi cohort and 8 deaths occurred within 150.7 pyrs in the RTX cohort. The respective all-cause mortality rates per 1000 pyrs were 94.8 (74.7-118.7) and 53.0 (22.9104.6). The unadjusted 5-year risk of mortality in the RTX-treated patients was approximately half that in the TNFi-treated patients, although this was not statistically 
Table 1 Baseline characteristics of the cohorts

\begin{tabular}{|c|c|c|c|}
\hline & RTX (n=43) & All TNFi $(n=309)$ & p Value \\
\hline Age (years) & $64.7(11.4)$ & $62.5(10.4)$ & 0.2 \\
\hline Female, n (\%) & $19(44.2)$ & 179 (57.9) & 0.09 \\
\hline \multicolumn{4}{|l|}{ Smoking history, $\mathrm{n}(\%)$} \\
\hline Current & 7 (16.3) & $64(20.7)$ & 0.6 \\
\hline Former & $27(62.8)$ & $169(54.7)$ & \\
\hline Never & $9(20.9)$ & 75 (24.6) & \\
\hline \multicolumn{4}{|l|}{ Ethnicity } \\
\hline White & $37(86.0)$ & $271(87.7)$ & 0.97 \\
\hline Other & $1(2.4)$ & 7 (2.3) & \\
\hline Missing, n (\%) & $5(11.6)$ & 31 (10.3) & \\
\hline DAS28 & $6.3(1.1)$ & $6.6(1.0)$ & 0.03 \\
\hline Missing, n (\%) & $0(0)$ & $2(0.6)$ & \\
\hline $\mathrm{HAQ}$ & $1.6(0.7)$ & $2.1(0.54)$ & $<0.001$ \\
\hline Missing, n (\%) & $6(13.9)$ & $20(6.4)$ & \\
\hline Disease duration (years), median (IQR)* & $5(3-13)$ & $12(7-20)$ & $<0.001$ \\
\hline Missing, $\mathrm{n}(\%)$ & $0(0)$ & $1(0.3)$ & \\
\hline Baseline steroid use, $\mathrm{n}(\%)$ & $27(62.8)$ & $177(57.3)$ & 0.5 \\
\hline Number of prior csDMARDs & $3.2(1.2)$ & $4.0(1.6)$ & $<0.01$ \\
\hline \multicolumn{4}{|l|}{ Comorbidity, n (\%)† } \\
\hline None & $10(23.3)$ & $103(33.3)$ & 0.3 \\
\hline 1 comorbidity & $14(32.6)$ & $113(36.7)$ & \\
\hline 2 comorbidity & $13(30.2)$ & $64(20.7)$ & \\
\hline$\geq 3$ comorbidity & $6(13.9)$ & $29(9.4)$ & \\
\hline Hypertension & $18(42.9)$ & $123(39.9)$ & 0.7 \\
\hline Ischaemic heart disease (angina $+\mathrm{MI}$ ) & 9 (20.9) & $39(12.6)$ & 0.1 \\
\hline Stroke & 5 (11.9) & $16(4.9)$ & 0.09 \\
\hline Renal & $2(4.6)$ & $11(3.6)$ & 0.7 \\
\hline Diabetes & $4(9.3)$ & $17(5.6$ & 0.3 \\
\hline Liver disease & $1(2.3)$ & $12(3.9)$ & 0.7 \\
\hline Depression & $8(18.6)$ & $49(16.4)$ & 0.6 \\
\hline Lung (asthma + COPD) & $18(41.9)$ & $80(25.6)$ & 0.03 \\
\hline Asthma & 5 (11.9) & $32(10.4)$ & 0.8 \\
\hline Missing, n (\%) & $1(2.3)$ & $6(1.9)$ & \\
\hline COPD & 14 (33.3) & $58(18.8)$ & 0.03 \\
\hline Missing, $\mathrm{n}(\%)$ & $1(2.3)$ & 7 (2.3) & \\
\hline \multicolumn{4}{|l|}{ Year of registration $\ddagger$} \\
\hline$<2008$ & $0(0)$ & 309 (100) & NA \\
\hline$\geq 2008$ & $43(100)$ & $0(0)$ & \\
\hline
\end{tabular}

Values are mean (SD), unless otherwise stated; categorical variables compared using Pearson chi-squared tests and continuous variables compared using Student's t-tests; information about missing data only reported for variables in which missingness occurred.

${ }^{*}$ Comparison conducted using Wilcoxon rank-sum test.

†Hypertension, coronary heart disease (Ml or angina), stroke, lung (asthma or bronchitis/emphysema), diabetes mellitus, depression, renal disease and liver disease.

‡Due to differences in recruitment periods for cohorts, between group comparisons are not appropriate for year of registration. COPD, chronic obstructive pulmonary disease; csDMARDs, conventional synthetic disease modifying antirheumatic drug; DAS28, disease activity score-28; HAQ, Health Assessment Questionnaire; MI, myocardial infarction; pyrs, person years; NA, not applicable; RTX, rituximab; TNFi, tumour necrosis factor inhibitor. 


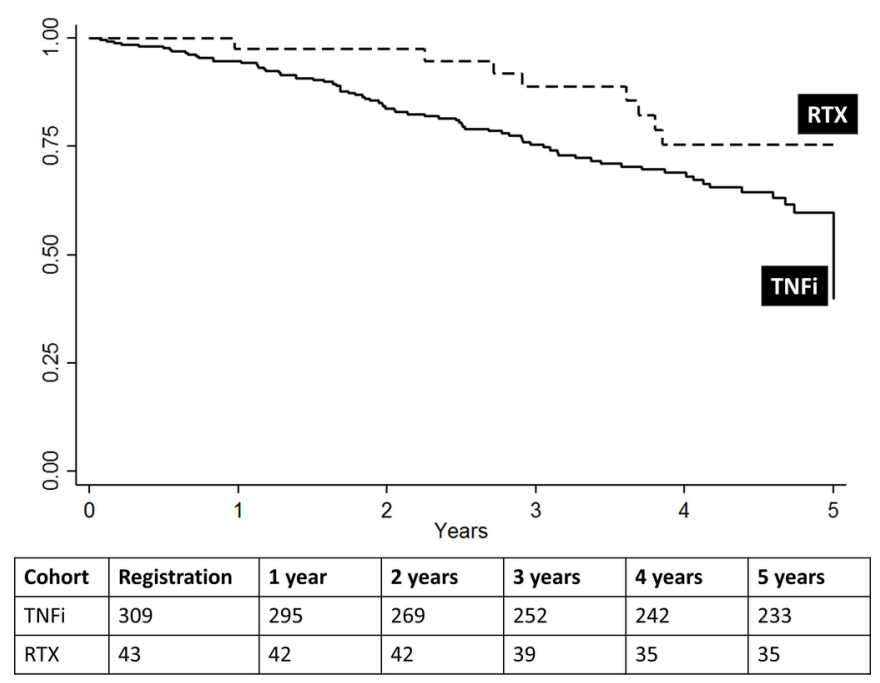

Figure 1 Kaplan-Meier survival curves for death following exposure to TNFi or RTX over the first 5 years following therapy commencement, within an intention to treat analysis. Numbers in table represent the number of patients at risk of death at the specific follow-up time points. RTX, rituximab; TNFi, tumour necrosis factor inhibitor.

significant (HR RTX compared with TNFi: $0.53,95 \%$ CI: 0.26 to 1.10 ; figure 1 ).

In addition to the a priori selected confounders, HAQ and DAS28 were also identified as significant confounders. After adjustment, the 5-year risk of mortality observed among patients starting RTX compared with TNFi remained reduced, but non-significant (0.49, 0.23 to 1.06 ).

Death certificate data were available for $75(98.7 \%)$ and $7(87.5 \%)$ of the deceased patients in the TNFi and RTX cohorts, respectively. Death certificates were unavailable for two patients (one in each cohort) who died outside the UK.

In total, 12 (16\%) 12 (16\%) TNFi deaths and 1 (14\%) RTX deaths had RA-ILD listed as the underlying cause of death (table 2). However, RA-ILD was more commonly reported anywhere on the death certificates of the RTX participants $(\mathrm{n}=5,71.4 \%)$ than those treated with TNFi $(\mathrm{n}=27,36.5 \%)$.

\section{DISCUSSION}

Patients with physician-recorded RA-ILD who received RTX as their first biologic for RA appeared to have better long-term survival compared with patients who received TNFi. This finding persisted after adjusting for key markers of RA disease severity. The proportion of deaths attributed to RA-ILD did not differ between the two groups.

This study has a number of limitations. First, these are observational data and the impact of non-randomisation to treatment is confounded by the fact that the two cohorts of patients included in this analysis were recruited over different time frames, where the approach to diagnosis and management of RA, and therefore possibly mortality, have differed. Although differences in disease severity and duration were identified and controlled for in our analysis, the early period of recruitment for TNFi also included a time prior to any guidelines regarding biologic treatment and RA-ILD, while later periods of recruitment may span a time when, in response to guidelines, patients with the most severe ILD may not have been prescribed a biologic at all. In part, these differential periods of recruitment were driven by the overall design of the BSRBR-RA, but there were 2 years of recruitment overlap during which no patients recruited to the register starting a TNFi had a history of RA-ILD.

Second, although this represents one of the largest outcome studies of RA-ILD in biologic-treated patients, including comprehensive drug exposure data and outcome data, the sample size, especially of patients exposed to RTX, was low, which may have affected power and also our ability to adjust further for other potential confounders using propensity scores.

Though different from a typical cohort of patients with RA starting a biologic, those included in this analysis were reflective of patients with RA-ILD, ${ }^{14}$ increasing the external validity of these findings. Unfortunately, data regarding the subtype or severity of the underlying RA-ILD, which have been associated with differences in mortality, were not available. It is recognised that prognosis is worse among patients with RA with usual interstitial pneumonia compared with those with non-specific interstitial pneumonia ${ }^{1420}$ and that one of the strongest predictors of mortality is pulmonary function. ${ }^{21}$ Therefore, the data presented cannot be used to make firm conclusions regarding relative safety of RTX and TNFi. Similarly, smoking data were not recorded over time and it would be beneficial to replicate this analysis in a cohort which included longitudinal data on ILD severity and smoking data.

Although it was not possible to establish whether the progression of the pulmonary disease was altered by the biologic agent, reassuringly, most of the deaths were not attributed to ILD. In previous analyses, a higher proportion of deaths were attributed to RA-ILD in patients commencing TNFi compared with those using sDMARDs. ${ }^{15}$ Here, RA-ILD was identified as the underlying cause of death in 1 of 7 RTX deaths $(14 \%)$ and 12 of 76 TNFi deaths (16\%). Nevertheless, RA-ILD was listed more often on the death certificates of patients in the RTX cohort. This may reflect that RA-ILD may have been a prominent feature that informed biologic choice among patients starting RTX.

In summary, this study provides some evidence to suggest that patients with RA with ILD who received RTX as their first biologic experienced better long-term survival compared with patients starting TNFi. However, the high proportion of death certificates on which ILD was present in the RTX cohort, the lack of information on severity or subtype of ILD and differences in time of recruitment precludes firm conclusions about comparative drug safety in this population. 
Table 2 Mortality rates and cause of death in patients with ILD treated with first-line TNFi or RTX in an intention to treat analysis

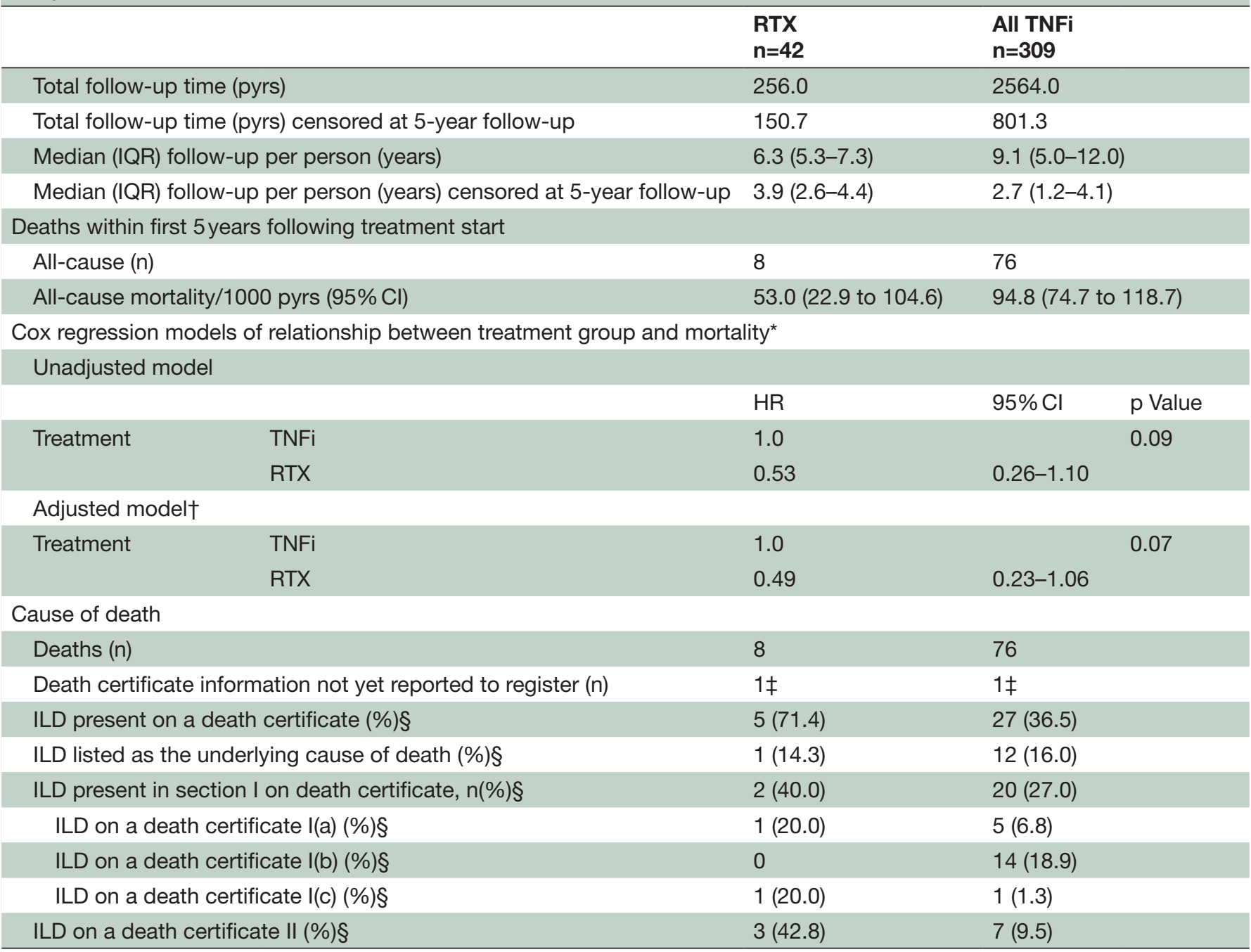

${ }^{*}$ Analysis conducted using multiple imputation for missing data.

†Age, disability (HAQ), disease activity (DAS28), disease duration and sex adjusted.

†Death occurred outside of the UK and therefore no death certificate and the cause of death cannot be determined.

§Percentage of those with death certificate data available.

DAS28, disease activity score-28; ILD, interstitial lung disease; HAQ, Health Assessment Questionnaire; pyrs, person years; RTX, rituximab;

$\mathrm{TNFi}$, tumour necrosis factor inhibitors.

Acknowledgements The authors acknowledge the enthusiastic collaboration of all consultant rheumatologists and their specialist nurses in the UK in providing the data (visit www.bsrbr.org for a full list of contributors). The authors would like to gratefully acknowledge the support of the National Institute for Health Research, through the Comprehensive Local Research Networks at participating centres. In addition, the authors acknowledge support from the BSR Executive, the members of the BSRBR Registers Committee and the BSRBR Project Team in London for their active role in enabling the register to undertake its tasks. The authors also acknowledge the seminal role of the BSR Clinical Affairs Committee for establishing national biological guidelines and recommendations for such a register. Finally, the authors would like to acknowledge the Arthritis Research UK Centre for Epidemiology that provided the infrastructure support for the study. An abstract based on this work was presented at the American College of Rheumatology 2016 ACR/ARHP Annual Meeting and was published as a conference abstract.

Contributors KLD, KI, KLH and CK: conception of design of the work. KW, DPMS and KLH: data collection. KLD, KLH and CK: data analysis and interpretation. KLD: drafting the article. KI, KW, DPMS, KLH and CK: critical revision of the article. All authors: final approval of the version to be published.

Funding This work was supported by the British Society for Rheumatology (BSR). The BSR commissioned the BSR Biologics Register in rheumatoid arthritis (BSRBR-RA) as a UK wide national project to investigate the safety of biologic agents in routine medical practice. KH is the principal investigator. BSR receives restricted income from UK pharmaceutical companies, including Abbvie, Celltrion, Hospira, MSD, Pfizer, SOBI, Samsung, UCB and Roche. This income finances a wholly separate contract between the BSR and the University of Manchester. The principal investigator and the BSRBR-RA team at the University of Manchester have full academic freedom and are able to work independently of pharmaceutical industry influence. All decisions concerning analyses, interpretation and publication are made autonomously of any industrial contribution. Members of the BSRBR-RA University of Manchester team, BSR trustees, committee members and staff complete an annual declaration in relation to conflicts of interest. All relevant information regarding serious adverse events outlined in the manuscript have been reported to the appropriate pharmaceutical company as per the contractual agreements/ standard operating procedures.

Competing interests None declared.

Patient consent Detail has been removed from this case description/these case descriptions to ensure anonymity. The editors and reviewers have seen the detailed information available and are satisfied that the information backs up the case the authors are making.

Ethics approval North West Multi-centre Research Ethics Committee (MREC 00/8/53). 
Provenance and peer review Not commissioned; externally peer reviewed.

Open Access This is an Open Access article distributed in accordance with the Creative Commons Attribution Non Commercial (CC BY-NC 4.0) license, which permits others to distribute, remix, adapt, build upon this work non-commercially, and license their derivative works on different terms, provided the original work is properly cited and the use is non-commercial. See: http://creativecommons.org/ licenses/by-nc/4.0/

(C) Article author(s) (or their employer(s) unless otherwise stated in the text of the article) 2017. All rights reserved. No commercial use is permitted unless otherwise expressly granted.

\section{REFERENCES}

1. Nannini C, Ryu JH, Matteson EL. Lung disease in rheumatoid arthritis. Curr Opin Rheumatol 2008;20:340-6.

2. Anaya JM, Diethelm L, Ortiz LA, et al. Pulmonary involvement in rheumatoid arthritis. Semin Arthritis Rheum 1995;24:242-54.

3. Hakala M. Poor prognosis in patients with rheumatoid arthritis hospitalized for interstitial lung fibrosis. Chest 1988;93:114-8.

4. Olson AL, Swigris JJ, Sprunger DB, et al. Rheumatoid arthritisinterstitial lung disease-associated mortality. Am J Respir Crit Care Med 2011;183:372-8.

5. Ledingham J, Deighton C, British Society for Rheumatology Standards, Guidelines and Audit Working Group. Update on the British Society for Rheumatology guidelines for prescribing TNFalpha blockers in adults with rheumatoid arthritis (update of previous guidelines of April 2001). Rheumatology 2005;44:157-63.

6. Gochuico BR, Avila NA, Chow CK, et al. Progressive preclinical interstitial lung disease in rheumatoid arthritis. Arch Intern Med 2008;168:159-66

7. Ramos-Casals M, Brito-Zerón P, Soto MJ, et al. Autoimmune diseases induced by TNF-targeted therapies. Best Pract Res Clin Rheumatol 2008;22:847-61.

8. Keir GJ, Maher TM, Ming D, et al. Rituximab in severe, treatmentrefractory interstitial lung disease. Respirology 2014;19:353-9.

9. Bukhari M, Abernethy R, Deighton C, et al. BSR and BHPR guidelines on the use of rituximab in rheumatoid arthritis. Rheumatology 2011;50:2311-3.
10. Burton C, Kaczmarski R, Jan-Mohamed R, et al. Interstitial pneumonitis related to rituximab therapy. $N$ Engl $\mathrm{J} \mathrm{Med}$ 2003;348:2690-1.

11. Naqibullah M, Shaker SB, Bach KS, et al. Rituximab-induced interstitial lung disease: five case reports. Eur Clin Respir $J$ 2015;2:27178

12. Jani M, Hirani N, Matteson EL, et al. The safety of biologic therapies in RA-associated interstitial lung disease. Nat Rev Rheumatol 2014;10:284-94.

13. Matteson EL, Bongartz T, Ryu JH, et al. Open-Label, pilot study of the safety and clinical effects of Rituximab in patients with rheumatoid Arthritis-Associated interstitial pneumonia. Open $J$ Rheumatol Autoimmune Dis 2012;02:53-8.

14. Iqbal K, Kelly C. Treatment of rheumatoid arthritis-associated interstitial lung disease: a perspective review. Ther Adv Musculoskelet Dis 2015;7:247-67.

15. Dixon WG, Hyrich KL, Watson KD, et al. Influence of anti-TNF therapy on mortality in patients with rheumatoid arthritisassociated interstitial lung disease: results from the British Society for Rheumatology Biologics Register. Ann Rheum Dis 2010;69:1086-91.

16. Watson K, Hyrich K. The role of the British Society for Rheumatology Biologics Register (BSRBR) and the NICE guidelines for anti-TNF therapy. Musculoskeletal Care 2003;1:58-64.

17. Watson K, Symmons D, Griffiths I, et al. The British Society for Rheumatology biologics register. Ann Rheum Dis 2005;64 Suppl 4:iv42-iv43.

18. Mäkinen $\mathrm{H}$, Kautiainen $\mathrm{H}$, Hannonen $\mathrm{P}$, et al. Is DAS28 an appropriate tool to assess remission in rheumatoid arthritis? Ann Rheum Dis 2005;64:1410-3.

19. Kirwan JR, Reeback JS. Stanford Health Assessment Questionnaire modified to assess disability in british patients with rheumatoid arthritis. Br J Rheumatol 1986;25:206-9.

20. Kelly $\mathrm{C}$, lqbal $\mathrm{K}$, Iman-Gutierrez L, et al. Lung involvement in inflammatory rheumatic diseases. Best Pract Res Clin Rheumatol 2016;30:870-88.

21. Assayag D, Lubin M, Lee JS, et al. Predictors of mortality in rheumatoid arthritis-related interstitial lung disease. Respirology 2014;19:493-500. 